\title{
Research on Green Process Optimization Method based on Local Optimi- zation of Process Feature
}

\author{
Song Yuchuan*, Zhou Jianbin and Lei Qi
}

State Key Laboratory of Mechanical Transmission, Chongqing University, Chongqing, 400030, China

\begin{abstract}
The green process was a necessary research field of the green manufacturing, which has drawn increasing attention from many scholars. This paper proposes a method to optimize green process routes based on local optimization of process feature and establish the green process databases for specific product range for the purpose of promoting the practicability of green process. For a range of products, the process features and process schemes are first focused on to build green process databases to support the process route optimization. Then with Leonid Potter interaction matrix model, each process route in the original set is evaluated separately and the process routes to be optimized and the "problem" processes are located. Later a model of optimizing the process elements is established to conduct the local optimization of the "problem" processes based on process features. Ultimately the optimal green process route is selected from the set of the optimized process routings. By an example, the method is verified to be feasible and efficient.
\end{abstract}

Keywords: Process planning, database, evaluation, optimization.

\section{INTRODUCTION}

Green process optimization oriented to the manufacturing process is the key to green manufacturing and the aim is to improve the green property of the manufacturing process by conducting optimization on process elements, process schemes and so on [1-3]. Currently, a number of studies on green process optimization have been carried out at home and abroad, including green process optimization strategies, green process databases, and green evaluation of process routes [4-7]. The research mentioned above has made a lot of achievements in basic theory, but in the practical applications there still exist the following difficulties: (1) Lack of available green process data standards to support green process research. In practice, it's a very difficult task to construct the green process databases for all the typical machining process. (2) The current green process optimization is generally only focused on a specific process element, and the methods to select the optimal process route are also limited and one-sided [8-12]. Therefore, how to establish practical green process databases, and further to conduct a comprehensive and efficient green process optimization is the focus of the study.

The paper, from the practical aspect, first establishes the green process databases for a key product range. Then, on the foundation of the process routes being used in practice, the green process optimization based on local optimization of process features is conducted with the support of green process databases, following the steps of evaluation, local optimization, and decision-making. Because of the full use of the existing process routes that has been proved to be feasible in production practice, the method could improve the green property of manufacturing process with great efficiency and strong practicability.

\section{THE ARCHITECTURE OF GREEN PROCESS OPTIMIZATION METHOD BASED ON LOCAL OPTIMIZATION OF PROCESS FEATURE}

Green process optimization is mainly about making green auxiliary decision based on traditional process planning. The environmental friendliness of manufacturing process could be improved through optimizing process schemes, process equipment and process parameters. In order to support green process optimization, the practical green process databases for a specific range of products needs necessarily to be built by considering the process schemes of each process feature and the related process resources. On the basis of the IPO process, each available process rout is separately evaluated and analyzed with fuzzy comprehensive evaluation and Leon Potter interaction matrix to determine the process routes and the "problem" processes to be optimized. Then the model of local process elements optimization based on process feature is established to improve the green property of the process routes obtained above. Finally, the optimal process route is selected from the optimized process route set. The architecture of green process optimization method based on local optimization of process feature consists of five aspects, as shown in Fig. (1)

The process routes being used have been proven by the production practice to be superior in some traditional indexes, such as time and quality. Different process routes mainly come from the change of process elements, such as equipment, knives, cutting fluids [13]. So, it is feasible and 


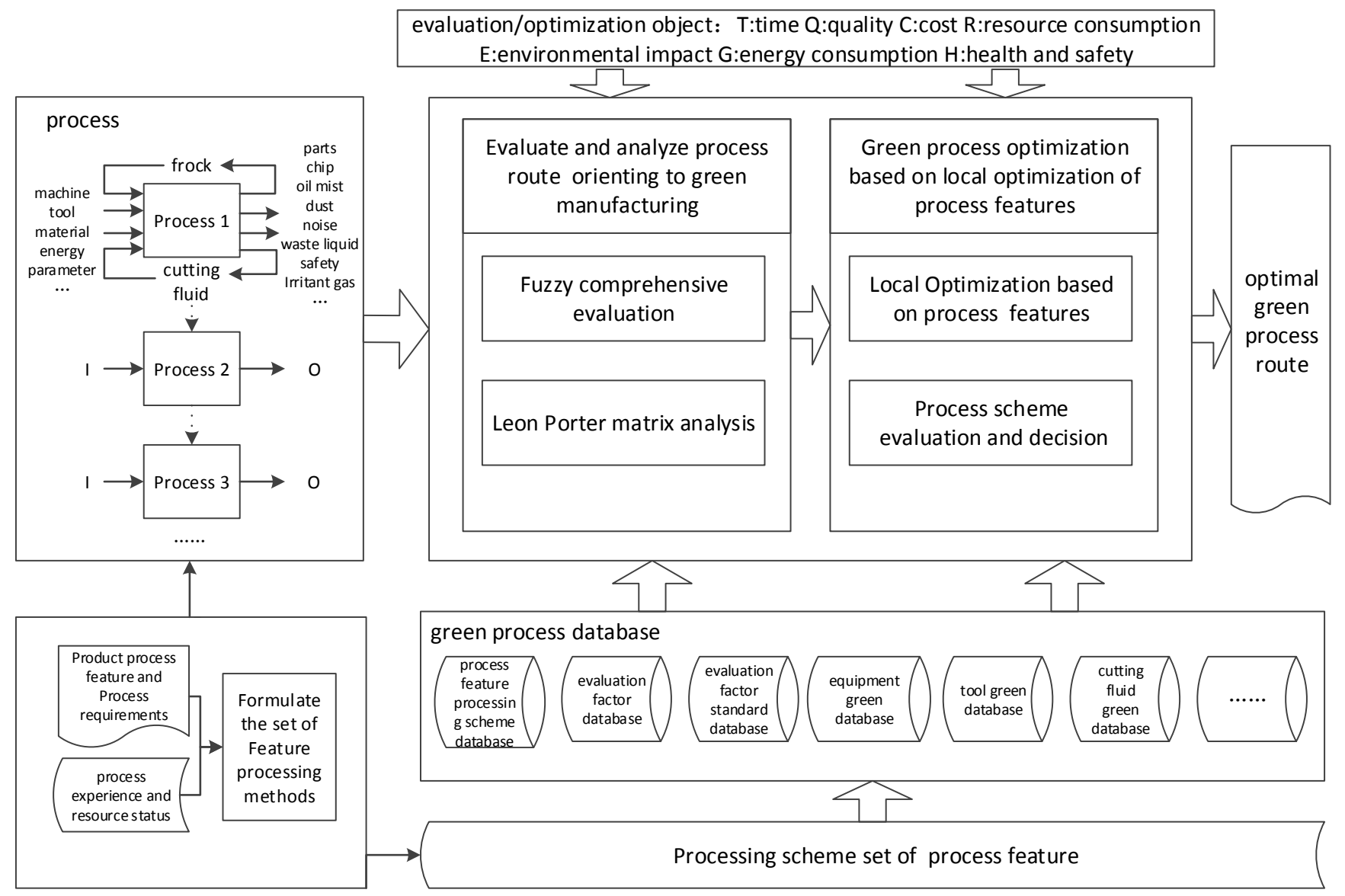

Fig. (1). The architecture of green process optimization method.

effective to locate the processes with poor green performance in process routes and conduct targeted local optimization based on process features on the premise of keeping the general machining sequence of the original process routes unchanged. Since the optimization is based on local process features, it is possible to optimize the poor indexes while retaining the good performances of the original process routes. In this way, the problems that may be caused by making totally new process routes could be avoided.

The procedures of the green process optimization based on local process feature optimization are shown in Fig. (2). Since the operation of the whole process need the support of green process databases, it is necessary to establish green process databases for the involved products.

\section{CONSTRUCTION OF GREEN PROCESS DA- TABASES FOR THE SPECIFIC PRODUCT RANGE}

The green process database is a set of data reflecting the environmental impact and resource consumption of the manufacturing process to support process route evaluation and green process optimization. Different from the traditional process planning, green process optimization concerns about not only the traditional characteristics of input and output, such as quality, cost, effectiveness, but also the environment and resource characteristics, namely the green property of the process elements. On the other hand, the other emissions outputs besides the products are also concerned and the factors that measure these outputs are defined as the green evaluation factors of the manufacturing process. Green process databases should be able to provide the green property knowledge of the process elements involved in the specific process steps and the green evaluation factors of the outputs to assist green process optimization and decision-making. Generally, the databases include three aspects: product features, process schemes and process routes databases; process element green databases and process evaluation factors databases, as shown in Fig. (3). The three green process databases specify the relationship between the input and the output so it could provide quantified knowledge for the evaluation and support each step of green process optimization, making the results more convincing.

\section{LOCAL OPTIMIZATION OF PROCESS ROUTES BASED ON PROCESS FEATURES}

\subsection{Evaluation of Process Routes}

According to the connotation of green manufacturing, green evaluation system consists of a set of green indexes including environmental impact, resources consumption, and 


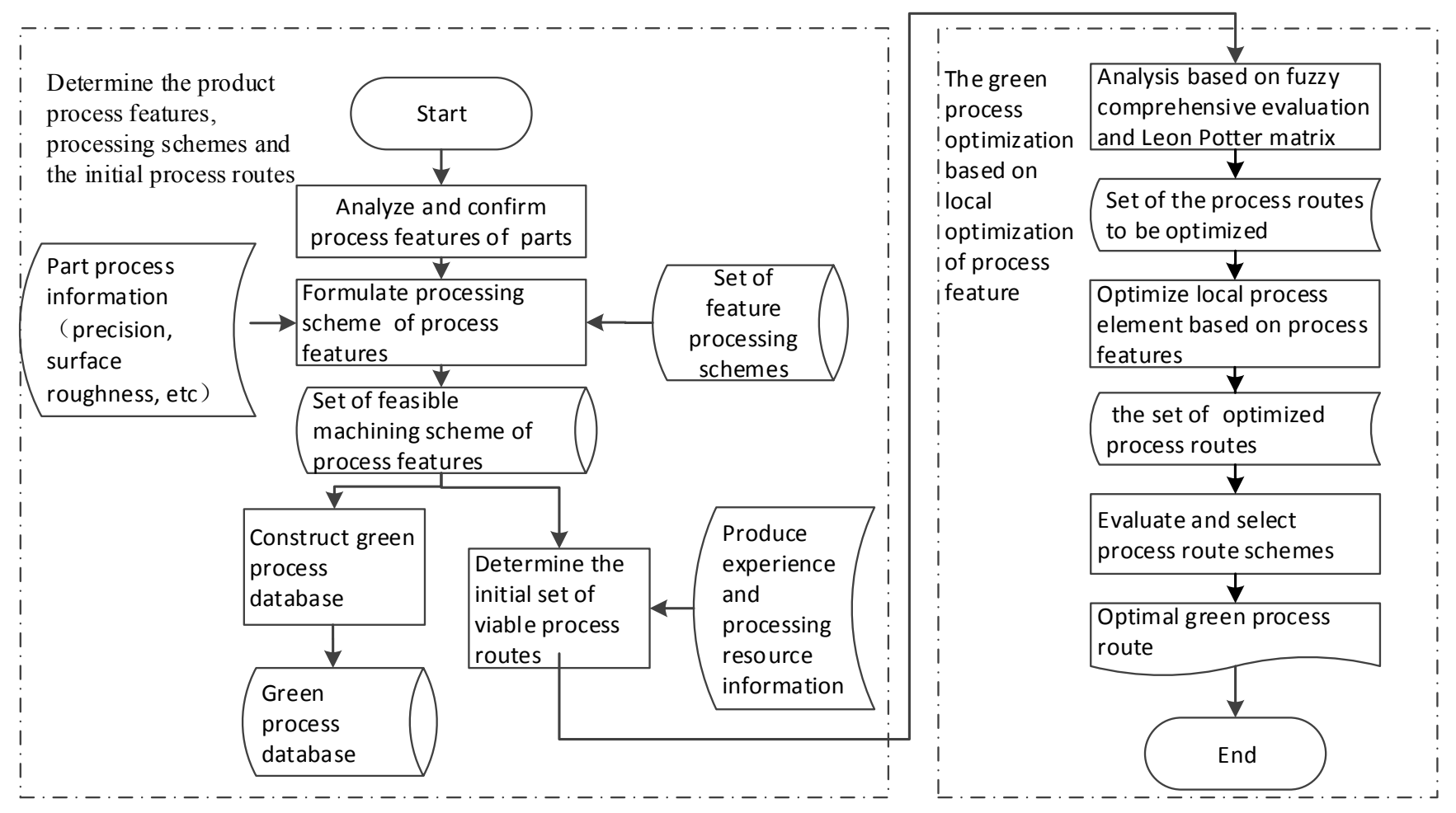

Fig. (2). Procedures of the green process optimization based on local optimization of process feature.

social benefits. Indexes of all aspects should be decomposed and refined to lower layer until the bottom layer of green evaluation factors, making up a multi-level, comprehensive and quantifiable evaluation index system. Values of the indexes in each layer are closely related to the specific process and could be quantified from the lower layer through comprehensive calculation. Since the green properties of inputs (including materials, equipment, tools, cutting fluids, process methods, etc.) are different and interacted with each other, the outputs would be different. Furthermore the values of evaluation indicators would be affected. Therefore, green process databases could provide the knowledge of the green properties of all process resources to support the green evaluation of process routes and to assist in the decision-making on selecting the process resources during process optimization.

The first-level comprehensive index set $I$ could be got by adding green indicators including resources $R$, environment $E$, energy consumption $G$, health and safety $H$ to traditional indexes of process planning including time $T$, quality $Q$, and $\operatorname{cost} C$. So $I=\{t, q, c, r, e, g, h\}$. Then with analytic hierarchy process (AHP), the weight of each index could be determined to obtain the weight vector $W_{F}=\left[\begin{array}{lllllll}\omega_{t} & \omega_{q} & \omega_{c} & \omega_{r} & \omega_{e} & \omega_{g} & \omega_{h}\end{array}\right]$. Because the process routes could be viewed as a chain composed of a series of IPO processes, the evaluation results of process routes could be calculated by evaluating these individual IPO processes and integrating the obtained results.
Suppose that a process route consists of $\mathrm{n}$ processes and $U_{F j}=\left\{u_{1}, u_{2}, \mathrm{~L}, u_{j}, \mathrm{~L}, u_{n}\right\}$ is the process set. The evaluation of process routes is realized through the evaluation of the process set. First determine the evaluation factors and the property values of each process. Then classify the evaluation factors into the corresponding evaluation indexes and in this way the first-level comprehensive index result $r_{i j}$ of each process could be obtained by the weighted sum of the property values from bottom to up through the evaluation index system, finally we get the matrix of evaluation results of all process routes $R$ :

$$
R=\left[\begin{array}{llllll}
r_{t 1} & r_{t 2} & \mathrm{~L} & r_{t j} & \mathrm{~L} & r_{t n} \\
r_{q 1} & r_{q 2} & \mathrm{~L} & r_{q j} & \mathrm{~L} & r_{q n} \\
r_{c 1} & r_{c 2} & \mathrm{~L} & r_{c j} & \mathrm{~L} & r_{c n} \\
r_{r 1} & r_{r 2} & \mathrm{~L} & r_{r j} & \mathrm{~L} & r_{r n} \\
r_{e 1} & r_{e 2} & \mathrm{~L} & r_{e j} & \mathrm{~L} & r_{e n} \\
r_{g 1} & r_{g 2} & \mathrm{~L} & r_{g 1} & \mathrm{~L} & r_{g n} \\
r_{h 1} & r_{h 2} & \mathrm{~L} & r_{h 1} & \mathrm{~L} & r_{h n}
\end{array}\right]
$$

After finishing the collection of the basic evaluation information, establish the Leon Potter interaction matrix model to analyze the performance of all the process routes, as shown in Fig. (4). Comprehensive analysis. 


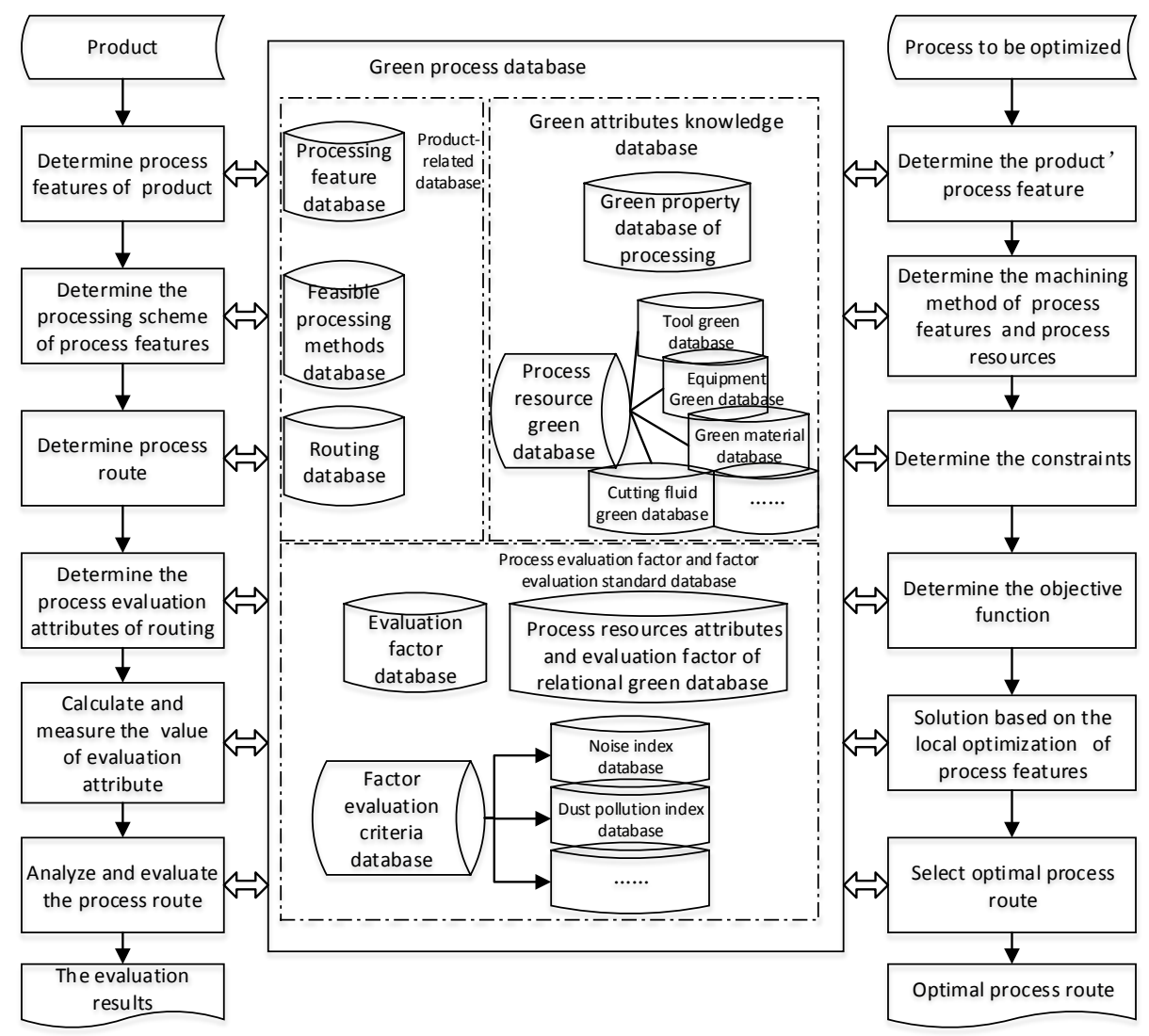

Fig. (3). Establish green process databases for specific product range.

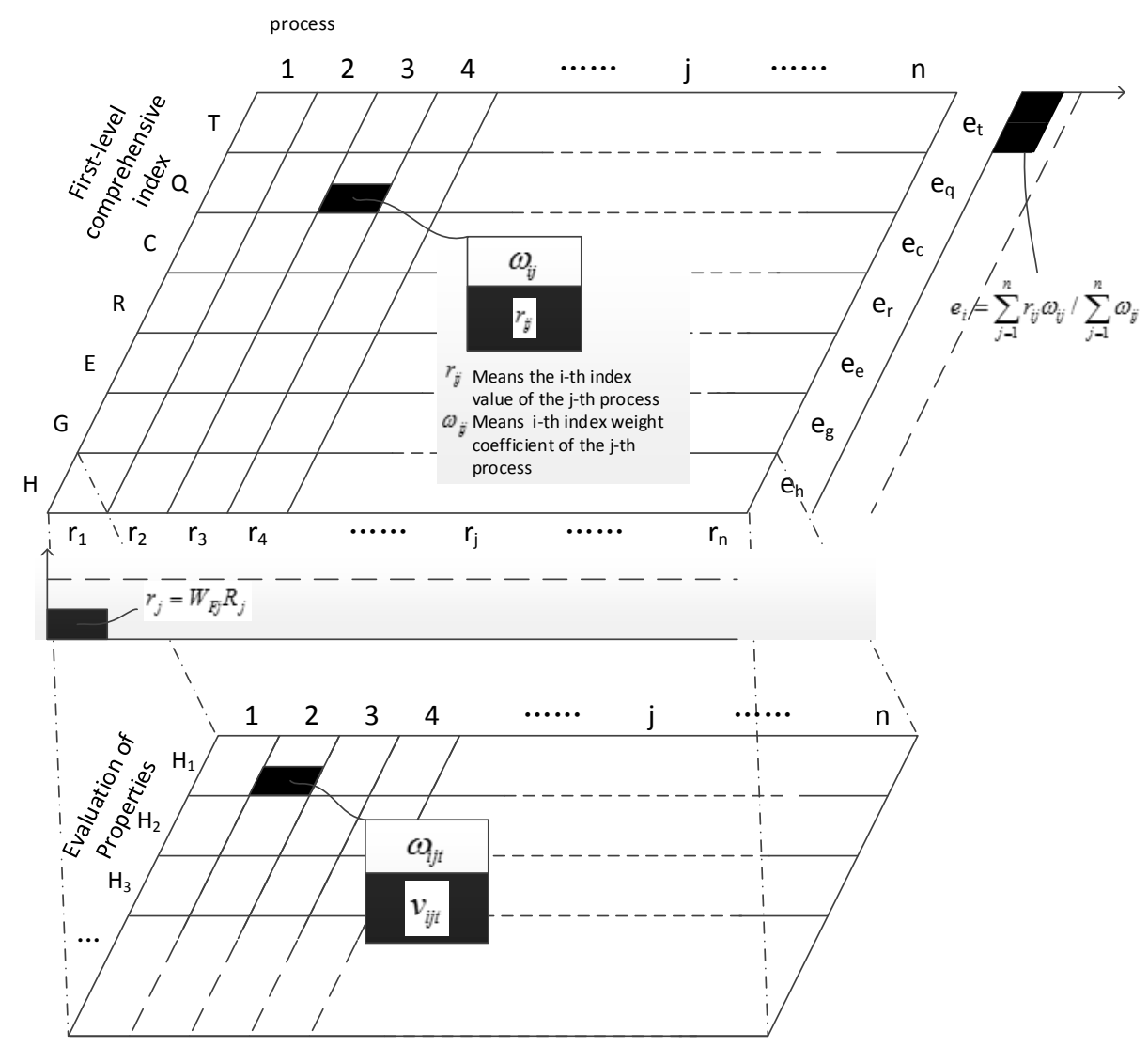

Fig. (4). Evaluation and analysis model based on the Leonid Potter interaction matrix. 


$$
E_{\text {com }}=\sum_{j=1}^{n} r_{j} / n
$$

$r_{j}=W_{F j} R_{j}$

$$
\begin{aligned}
& =\left[\begin{array}{lllllll}
\omega_{t j} & \omega_{q j} & \omega_{c j} & \omega_{r j} & \omega_{e j} & \omega_{g j} & \omega_{h j}
\end{array}\right] \\
& {\left[\begin{array}{lllllll}
r_{t j} & r_{q j} & r_{c j} & r_{r j} & r_{e j} & r_{g j} & r_{h j}
\end{array}\right]^{T}}
\end{aligned}
$$

$r_{j}$ denotes the comprehensive evaluation value of process $j$ and indicates its green performance. The comprehensive green property of process routes is calculated based on the comprehensive evaluation values of all its processes and the results will be regarded as the basis of selecting the optimal process route.

(2) Transverse analysis:

$$
E_{t r a}=\left[\begin{array}{lllllll}
e_{t} & e_{q} & e_{c} & e_{r} & e_{e} & e_{g} & e_{h}
\end{array}\right]^{T} e_{i}=\sum_{j=1}^{n} r_{i j} \omega_{i j} / \sum_{j=1}^{n} \omega_{i j}
$$

$e_{i}$ represents the evaluation value of the $i$ th first-level comprehensive index and $E_{\text {cro }}$ is the transverse index vector, based on which, the concave and convex condition of all the indexes could be obtained with the cluster analysis and the process routes with the similar distribution in $E_{c r o}$ are classified to the same category. Select the optimal process route in each category to compose the set of process routes to be optimized.

(3) Vertical analysis:

$$
E_{v e r}=W_{F} R=\left[\begin{array}{llll}
r_{1} & r_{2} & \mathrm{~L} & r_{n}
\end{array}\right]
$$

$E_{v e r}$ represents the vertical evaluation vector composed of the comprehensive evaluation value $r_{j}$ of each process. The processes with the relatively poor green performance in the process route could be located by the vertical comparison among all the processes according to their comprehensive evaluation values and the results would be referred as "problem" processes that will be focused on later as the object of the local optimization.

\subsection{Local Process Elements Optimization Model Based on Process Features}

After evaluating and analyzing process routes, the set of process routes and the processes to be optimized have been determined, and the green process optimization is transformed into local process elements optimization. The feasible process schemes of features and process elements resources involved are provided by the green databases to obtain the set of feasible process schemes of features. The local optimization model of process routes based on process features is established as follows:

(1)Variables Description
In the discussed issue, process features are taken as the optimization objects. The optimal scheme will be selected by determining the equipment $(e)$, tool $(t)$, blank $(b)$, cutting fluid $(f)$. Thus, the decision variable is:

$$
X=[e, t, b, f]^{T}
$$

\section{(2) Objective Functions}

In order to make the results more convincing, the optimization goals and the evaluation goals should be selected consistently. So time $(\mathrm{T})$, quality $(\mathrm{Q})$, cost $(\mathrm{C})$, resource consumption $(\mathrm{R})$, environmental impact $(\mathrm{E})$, energy consumption $(\mathrm{G})$, health $(\mathrm{H})$ are considered here.

i. The objective on $\mathrm{T}$ is to minimize the processing time of a single workpiece.

$$
\min f_{t}(X)=t_{c}+\frac{t_{c h}}{L}+t_{a}
$$

$t_{c}$-process cutting time; $t_{c h}$-tool changing time, including unloading tools, loading tools and setting tools; $L$-batch sizes; $t_{a}$-auxiliary time including loading and unloading, starting and stopping the machine etc.

ii. The objective on $\mathrm{Q}$ is to minimize the nonconforming rate:

$\min f_{q}(X)=Q=Q_{P}$

$Q$ - nonconforming rate.

iii. The objective on $\mathrm{C}$ is to minimize the cost of a single workpiece:

$$
\min f_{c}(X)=\left(t_{c}+\frac{t_{c h}}{L}+t_{a}\right)\left(M+C_{0}+C_{s}\right)+t_{c} \frac{C_{t}}{T}
$$

$M$-labor cost rate,

$C_{0}$-sharing rate of manufacturing overhead by work hours;

$C_{g}$-energy consumption rates,

$T$-tool life,

$C_{t}$-tool costs.

iv. The objective on $\mathrm{R}$ is to minimize total resource consumption of raw materials and auxiliary materials of a single workpiece. Raw material consumption is calculated by chipping allowance $R_{c a}$. Auxiliary materials include cutting fluid $R_{c f}$, fuel and other materials $R_{e l}$ :

$\min f_{r}(X)=R_{c a}+R_{c f}+R_{e l}=R_{c a}+\rho v_{c f} t_{c}+R_{e l}$ 
$\rho$-cutting fluid utilization,

$v_{c f}$-flow velocity of cutting fluid.

v.The objective on $\mathrm{E}$ is to minimize the comprehensive environmental pollution emissions:

$\min f_{e}(X)=g\left(E_{s}, E_{1}, E_{g}, E_{p h}\right)$

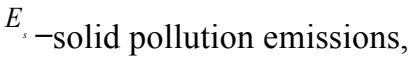

$E_{\imath}$-waste liquid emissions,

$E_{\mathrm{g}}$-waste gas emissions,

$E_{p h}$-physical emissions.

vi. The objective on $\mathrm{G}$ is to minimize the electricity consumption of a single workpiece:

$\min f_{g}(X)=a P_{c} t_{c}+P_{u}\left(\frac{t_{c h}}{L}+t_{a}\right)$

$P_{u}$-no-load power of machine,

$P_{c}$ _cutting power of machine,

$\alpha$-power balance equation coefficient, $\alpha=1.15-1.25$.

vii. The objective on $\mathrm{H}$ is weighed by the occupational safety factor and the effect of workshop's environmental pollution on workers' health.

$\min f_{h}(X)=H=\frac{H_{r}}{H_{m}}$

$H_{n}$-safety factor,

$H_{r}$-effect of workshop's environmental pollution on workers' health.

\section{(3) Constraint}

Green process optimization method based on local optimization of process features is operated on the basis of the process routes being used. The essence is to get the green process routes efficiently and quickly by optimizing local process elements. The constraints include the following three aspects:

i. Process requirements constraints. ii. Reasonability constraints. iii. Non-destroyed relationship constraints.

To sum up, the optimization model is:

$$
\begin{aligned}
& f(X)=\min \left[f_{t}(X), f_{q}(X), f_{c}(X), f_{r}(X), f_{e}(X), f_{g}(X), f_{h}(X)\right]^{T} \\
& X=[e, t, b, f]^{T} \\
& \text { s.t. } X \in D
\end{aligned}
$$

$D$ is the constraint set.

\subsection{A Model Solution Based on Ant Colony Algo- rithm}

The discussed issue is a multi-objective decision-making problem. The paper adopts ant colony algorithm to address it. As described above, a process feature generally requires a plurality of processing steps distributed in different processes. When a work step of a process feature is optimized, the work steps of this feature in other processes and the other features in this process should also be considered. In order to describe the relationships better, firstly, some variables are described and defined as follows:

$$
\begin{aligned}
& F=\left\{f_{1}, f_{2}, \mathrm{~L}, f_{n}\right\} \text { denotes the set of process features. } \\
& P=\left\{p_{1}, p_{2}, \mathrm{~L}, p_{n}\right\} \text { denotes the initial process route of }
\end{aligned}
$$
the product, and $p_{i}$ denotes the $i$ th process.

$P^{*}=\left\{p_{1}^{*}, p_{2}^{*}, \mathrm{~L}\right\}$ denotes the set of the "problem" processes to be optimized.

$F^{*}=\left\{f_{1}^{*}, f_{2}^{*}, \mathrm{~L}\right\}$ denotes the set of all the process features processed in the "problem" processes.

Definition 1 Work steps sequences of the feature: The process of the feature $f_{i}$ generally need several processing steps, and all these processing steps are defined as the work steps sequences of $f_{i}$, expressed as $S_{i}=\left[\begin{array}{llll}s_{i 1} & S_{i 2} & \mathrm{~L} & S_{i m}\end{array}\right] \cdot s_{i j}$ denotes the $j$ th work step of feature $f_{i}$, and $m$ denotes the total number of the work steps.

Definition 2 Work steps related in the same process: Different process features may be machined in the same process. The involved work steps of these process features are called work steps related in the same process.

Definition 3 Work step nodes: There are many process resource combinations to complete the process task of work step $s_{i j}$. These combinations are the variables of the local optimization and the basic move nodes of ant colony algorithm. They are defined as work step nodes and expressed as $o_{i j x}=\{e, t, b, f\}$.

Definition 4 Work step node unit: All the work step nodes of work step $s_{i j}$ make up the work step node unit of $S_{i j}$

$O_{i j}=\left\{o_{i j 1}, o_{i j 2}, \mathrm{~L}, o_{i j n}\right\}$.

According to the principle of ant colony algorithm, work step nodes are regarded as the ants' traveling nodes. So, the solution based on ant colony algorithm could be described as: Based on the process features of the "problem" process in the initial process route, ants move among the work step nodes of these features to find the optimal work steps sequence that is essentially made up by the optimal process resource combination of each feature. 


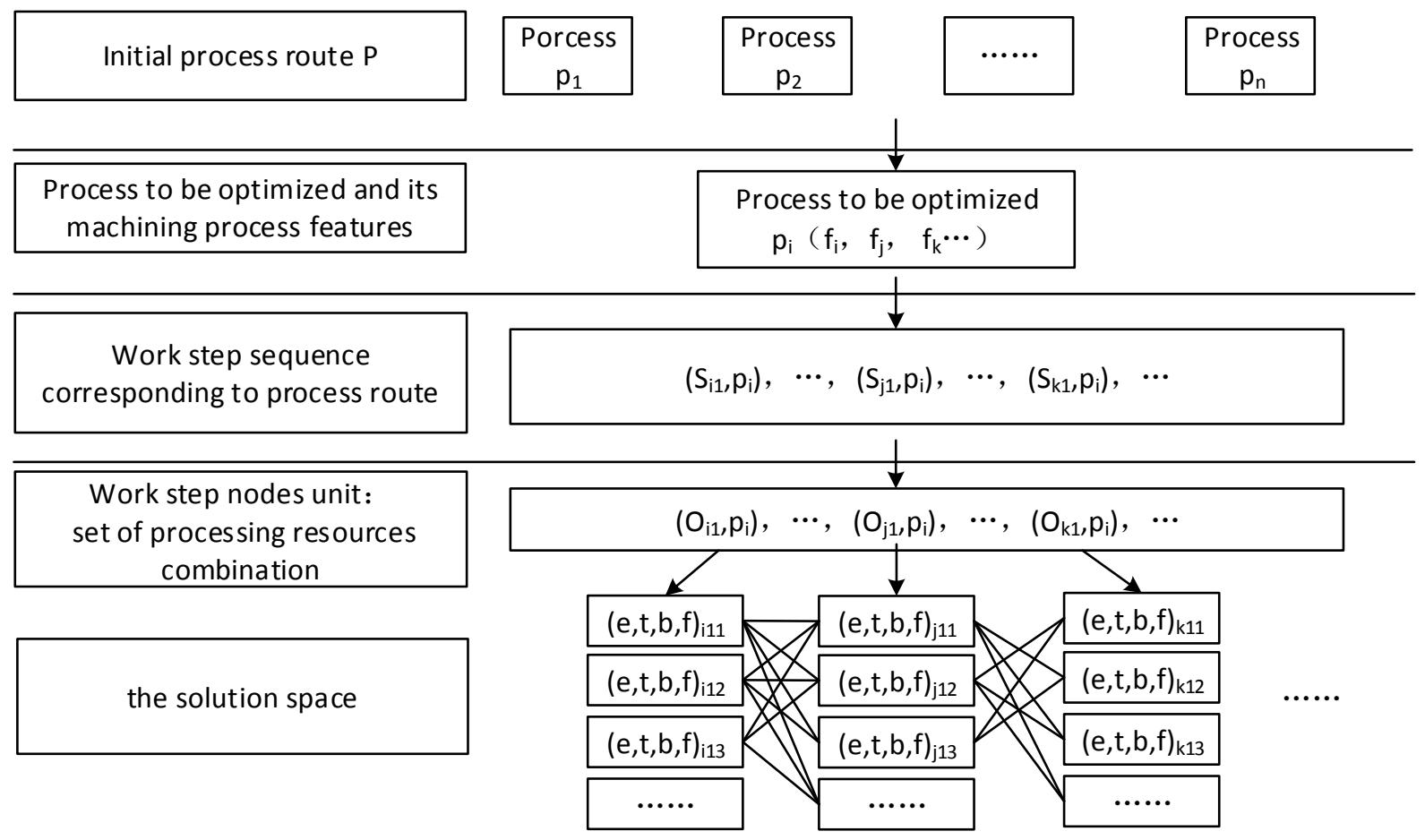

Fig. (5). Solution space of process route optimization.

The work steps sequence to be optimized originates from two aspects: one is all the work steps of the process feature in "problem" process, and the other is the work steps related in the same process. Work step $s_{i j}$ is expressed as $\left(s_{i j}, p_{m}\right)$ and $p_{m}$ is the process that $s_{i j}$ is located in the initial process routes. In this way, the work steps sequence to be optimized could be expressed as $\left\{\left(s_{i 1}, p_{m}\right), \mathrm{L}\left(s_{j 1}, p_{n}\right), \mathrm{L}\left(s_{k 1}, p_{o}\right), \mathrm{L}\right\}$. All the work step node units constitute the optimal solution space $O$.

$$
\begin{aligned}
& o=\left\{\left(S_{f 1}, P_{m}\right), \ldots\left(S_{f 1}, P_{n}\right), \ldots\left(S_{f 1}, P_{o}\right), \ldots\right\} \\
& =\left\{\left(O_{f 1}, P_{m}\right)_{, \ldots} .\left(O_{f 1}, P_{n}\right), \ldots\left(O_{f 1}, P_{o}\right), \ldots\right\} \\
& {\left[\left\{\begin{array}{c}
(e, t, b, f)_{f 11} \\
(e, t, b, f)_{f 12} \\
(e, t, b, f)_{f 13} \\
\ldots
\end{array}\right\}_{P_{m}} \ldots\left[\begin{array}{c}
(e, t, b, f)_{f 11} \\
(e, t, b, f)_{f 12} \\
(e, t, b, f)_{f 13} \\
\ldots
\end{array}\right\}_{P_{n}} \quad \ldots\left[\begin{array}{c}
(e, t, b, f)_{f 11} \\
(e, t, b, f)_{f 12} \\
(e, t, b, f)_{f 13} \\
\ldots
\end{array}\right\}_{P_{o}}\right] \ldots}
\end{aligned}
$$

Fig. (5) shows the hierarchical relationship of the original process routes, the process to be optimized and the work step nodes of process feature.

The purpose of local optimization based on process feature is to find an optimal green process route among the work step nodes. Similar to the basic ant colony algorithm, the method regards the work step nodes as the basic optimization nodes of ants. Choosing different work step nodes leads to different paths and the comprehensive green property of different work step nodes is equal to the length of the different path. $O=\left\{\left(O_{i 1}, p_{m}\right), \cdots\left(O_{j 1}, p_{n}\right), \ldots\left(O_{k 1}, p_{o}\right), \ldots\right\}$ is the work step node unit. Because the local optimization is carried out on the basis of the initial process routes, so the sequence of the work steps have been established, and choosing the optimal work step nodes would follow this sequence. $R_{i j}=\left\{r_{i j 1}, r_{i j 2}, \mathrm{~L}\right\}$ is the set of the comprehensive green property of each work step node. $R=\left\{\left(R_{i 1}, p_{n}\right), \ldots\left(R_{j 1}, p_{n}\right), \ldots\left(R_{k 1}, p_{o}\right), \ldots\right\}$ is the set of the green evaluation results corresponding to the work step nodes. $G=(O, R)$ is a directed graph that contains the information of a specific work step sequence. The task is to find the optimal work step node of each work step based on the specific work step sequence from G. These optimal work step nodes constitute an optimal green process route. Then, according to the correspondence between each work step with the process in the original routing, the optimized process route would be finally obtained by changing the corresponding processes in the original routing.

\section{VERIFICATION IN EXAMPLES}

A specific mechanical part is selected for example to illustrate the effectiveness of the designed method.

\section{(1) Construction of the green process databases}

The green process databases for the product are established, including the green databases of the process features, the green databases of the feature processing schemes and the green databases of all the involved process resources. 


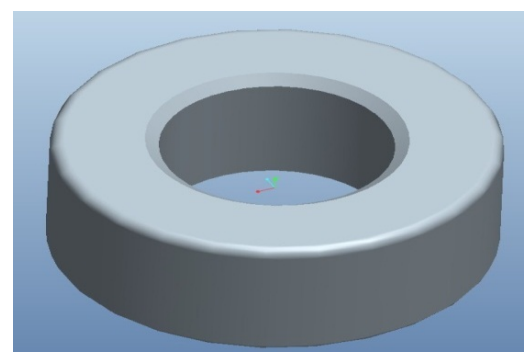

Fig. (6). Three-dimension graph of a part.

Table 1. Initial routings.

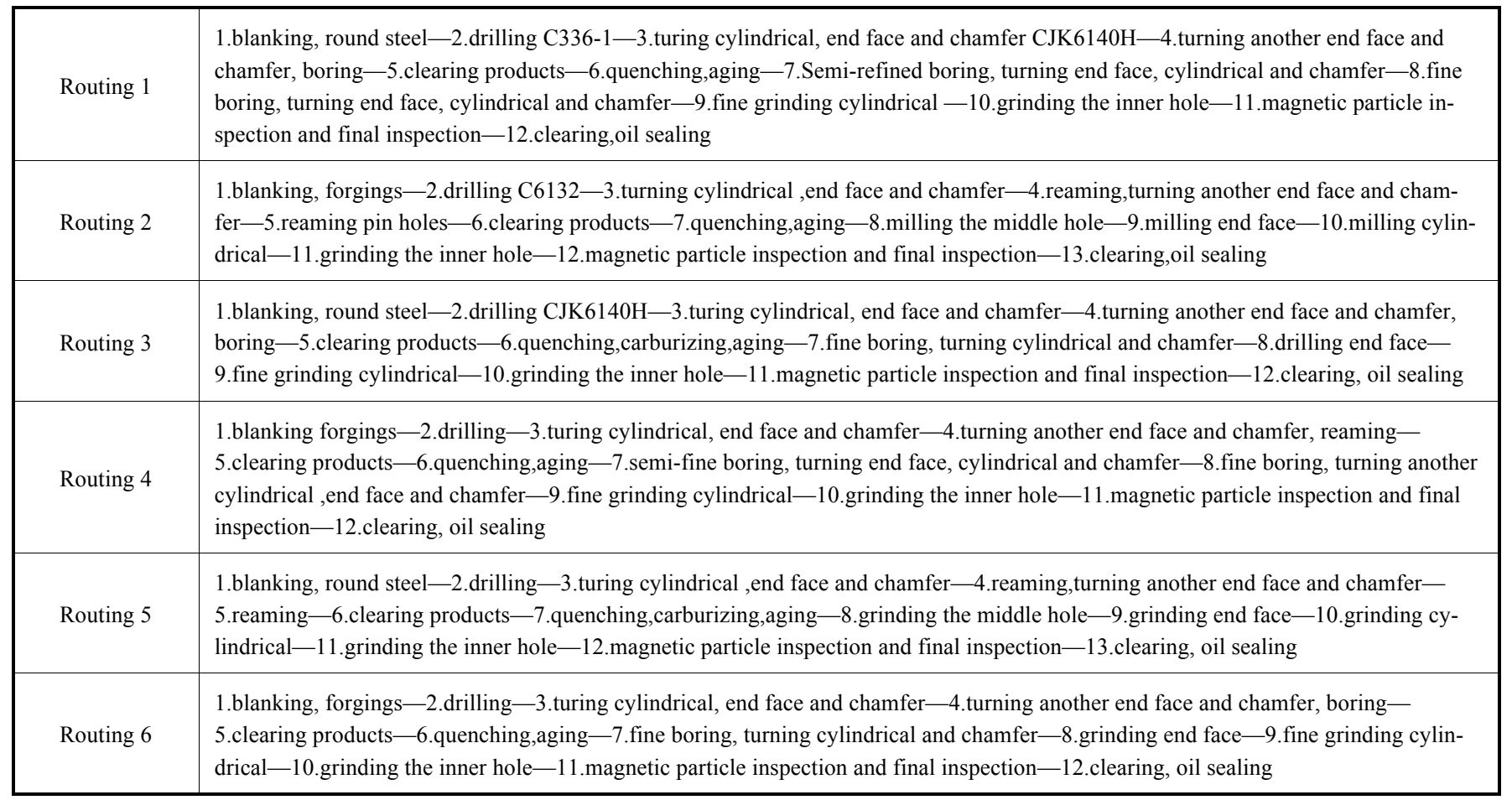

The three-dimensional model is shown in Fig. (6). The process features include two end faces, a cylindrical surface, a hole and two chamfers.

Each process feature has a variety of processing schemes. Based on the process requirements and the productivity of the enterprise, some feasible processing schemes are made for each process feature by the process department. For example, there are five schemes for the hole. $\mathrm{u} 1=\{$ drilling $\rightarrow$ expanding $\rightarrow$ reaming $\rightarrow$ grinding $\}$; $\mathrm{u} 2=\{$ drilling $\rightarrow$ expanding $\rightarrow$ rough reaming $\rightarrow$ fine reaming $\}$; $\mathrm{u} 3=$ \{rude boring $\rightarrow$ semi-fine boring $\rightarrow$ fine boring $\}$; u4 $=$ \{drilling $\rightarrow$ rude expansion $\rightarrow$ fine expansion $\rightarrow$ hinging $\}$; u5 $=$ \{rude boring $\rightarrow$ semi-fine boring $\rightarrow$ grinding . They constitute the databases of the processing schemes for a hole. At the same time, the green databases of all the involved process resources could also be built.

\section{(2) Green process optimization}

Step1: Collect the initial process routes of the product, as shown in Table 1.
Step2: Employ fuzzy comprehensive evaluation and AHP to conduct comprehensive green property evaluation to the initial process routes. Take routing 1 for example: first determine the weights of the first-level comprehensive index with AHP and get the result $W_{F}=\left[\begin{array}{lllllll}0.153 & 0.207 & 0.198 & 0.116 & 0.142 & 0.124 & 0.060\end{array}\right]$. Then classify the green evaluation factors into the corresponding index and determine their weights also with AHP. Evaluate the value of each green evaluation factor with fuzzy evaluation method to obtain the evaluation result of the firstlevel comprehensive index, as shown in Tab 2

Similarly, evaluate the other initial process routes separately.

Step 3: Analyze the evaluation results with Leon Potter interaction matrix. First calculate the comprehensive evaluation results of each process route. Then, calculate the transverse evaluation results, based on which divide the initial process routes into two groups, as shown in Table 3 . 
Table 2. The evaluation results of the routing 1.

\begin{tabular}{|c|c|c|c|c|c|c|c|c|c|c|c|c|}
\hline First-level comprehensive index and weights & \multicolumn{12}{|c|}{ Evaluation value of processes } \\
\hline $\mathrm{Q}(0.207)$ & 96 & 98 & 98 & 98 & 100 & 92 & 98 & 98 & 99 & 95 & 100 & 97.45 \\
\hline $\mathrm{E}(0.116)$ & 80 & 93 & 90 & 90 & 94 & 96 & 95 & 95 & 92 & 87 & 86 & 90.73 \\
\hline $\mathrm{R}(0.142)$ & 89 & 94 & 94 & 93 & 90 & 94 & 94 & 95 & 89 & 81 & 83 & 90.55 \\
\hline $\mathrm{G}(0.124)$ & 92 & 96 & 94 & 93 & 99 & 95 & 92 & 93 & 89 & 98 & 98 & 94.45 \\
\hline $\mathrm{H}(0.060)$ & 95 & 92 & 94 & 93 & 90 & 94 & 95 & 95 & 86 & 80 & 84 & 90.73 \\
\hline
\end{tabular}

Table 3. Transverse index evaluation and analysis of process routings.

\begin{tabular}{|c|c|c|c|c|c|c|}
\hline & Routing 1 & Routing 2 & Routing 3 & Routing 4 & Routing 5 & Routing 6 \\
\hline Comprehensive evaluation value & 93.16 & 89.89 & 91.77 & 93.04 & 89.22 & 92.40 \\
\hline Q & 97.45 & 96.92 & 98.18 & 97.73 & 97.17 & 97.64 \\
\hline $\mathrm{R}$ & 90.73 & 91.17 & 88.09 & 91.82 & 88.83 & 90.45 \\
\hline $\mathrm{E}$ & 90.55 & 86.25 & 88.18 & 90.82 & 84.92 & 89.55 \\
\hline G & 94.45 & 90.08 & 92.64 & 93.55 & 89.67 & 93.27 \\
\hline $\mathrm{H}$ & 90.73 & 85.00 & 88.82 & 89.73 & 84.83 & 88.73 \\
\hline
\end{tabular}

The first group includes routing 1, 3, 4 and 6, and their performance on $\mathrm{T}, \mathrm{G}$ and $\mathrm{Q}$ are better; the second group includes routing 2 and 5 , and their performance on $\mathrm{Q}, \mathrm{R}$ and $\mathrm{G}$ are better. Choose the process routes with the better comprehensive evaluation results in each group as the process routes to be optimized. Here is routing 1 and 2 . It could be seen from Table 3 that all the process routes fail to perform well on $\mathrm{E}$ and $\mathrm{H}$. Through analyzing we find that process 10 is manual grinding, and process 11 is manual cleaning with gasoline, which would release a lot of waste liquid and gases and bring much harm to the workers and that is supposed to be the main reasons leading to the bad performance on $E$ and $\mathrm{H}$.

Calculate the comprehensive evaluation value of each process, and the processes whose comprehensive evaluation results are in the last $30 \%$ are selected as the objects of local optimization. The results are the 10th and 11th process of routing 1 and the 2 ed, 8 th and 9th process of routing 2 , as shown in Table 4.
Step 4: Optimize the process elements of the "problem" processes. Taking the 10th process of routing 1 for example, the process feature is inner hole and the processing step is the last fine operations. The work step nodes are found out (here only considering the process scheme and machine) from the corresponding green databases, as shown in Table $\mathbf{5}$. Through solving the optimization model, the obtained optimal processing scheme is grinding and the optimal processing equipment is honing machine. Thus, there is no need to smear lapping paste and grind manually. So, the performance on $\mathrm{E}$ and $\mathrm{H}$ will be largely improved. Similarly, the 11 th process of routing 1 is optimized into washing machine cleaning and cleaning fluid is placed by synthetic cleaner. The 2 nd process of routing 2 is optimized into drilling with the turret lathe C336-1. The 8th process is optimized into fine boring, and the 9th process is optimized into high speed fine milling with vertical machining center SV-1000A. Thus, the optimized process routings 1'and 2' are obtained, as shown in Table 6. 
Table 4. Longitudinal evaluation and analysis among processes in process routes to be optimized.

\begin{tabular}{|c|c|c|c|c|c|c|c|c|c|c|c|c|c|}
\hline \multirow{2}{*}{ Routings } & \multicolumn{12}{|c|}{ Longitudinal Evaluation Process Value } & \multirow{2}{*}{$\begin{array}{c}\text { Process Comprehensive } \\
\text { Value }\end{array}$} \\
\hline & 1 & 2 & 3 & 4 & 5 & 6 & 7 & 8 & 9 & 10 & 11 & 12 & \\
\hline Routing1 & 91.3 & 95.2 & 94.7 & 94.5 & 95.8 & 94.2 & 93.7 & 94.6 & 93.0 & 88.4 & 89.3 & - & 93.16 \\
\hline Routing2 & 90 & 87.3 & 89.6 & 89.3 & 92.5 & 95.8 & 94.2 & 82.2 & 87.2 & 92.9 & 88.4 & 89.3 & 89.89 \\
\hline
\end{tabular}

Table 5. The solution space of routing 1.

\begin{tabular}{|c|c|c|c|c|c|c|}
\hline $\begin{array}{c}\text { Work Steps } \\
\text { Sequence }\end{array}$ & $\left(s_{11}, p_{1}\right)$ & $\left(s_{21}, p_{2}\right)$ & $\left(s_{22}, p_{7}\right)$ & $\left(s_{23}, p_{8}\right)$ & $\left(s_{24}, p_{10}\right)$ & $\left(s_{31}, p_{11}\right)$ \\
\hline \hline \multirow{6}{*}{ Work step nodes } & Round steel & Drilling C6132 & $\begin{array}{c}\text { Semi-fine boring } \\
\text { CJK6140H }\end{array}$ & $\begin{array}{c}\text { Fine boring } \\
\text { SKT21 }\end{array}$ & $\begin{array}{c}\text { Grinding } \\
\text { M4515 }\end{array}$ & $\begin{array}{c}\text { Manual cleaning } \\
\text { with gasoline }\end{array}$ \\
\cline { 2 - 7 } & Forging & Drilling Z5180b & Reaming C6132 & $\begin{array}{c}\text { Hole grinding } \\
\text { M2110 }\end{array}$ & $\begin{array}{c}\text { Washing machine clean- } \\
\text { ing }\end{array}$ \\
\cline { 2 - 7 } & & Drilling C336-1 & & & & \\
\hline
\end{tabular}

Table 6. The optimized routings.

\begin{tabular}{|c|c|}
\hline Routing 1' & $\begin{array}{l}1 \text { blanking, round steel }-2 \text { drillingC } 336-1-3 \text { turning of outside round, face and chamfer }-4 \text { turning of other face and chamfer, bor- } \\
\text { ing }-5 \text { cleaning parts }-6 \text { quench-aging }-7 \text { semi-fine boring hole, turning of face ,outside round and chamfer }-8 \text { fine boring, turning } \\
\text { of other face ,outside round and chamfer }-9 \text { fine grinding outside round }-10 \text { inside hole honing }-11 \text { magnetic particle testing and final } \\
\text { checkout }-12 \text { washing machine cleaning, oil seal }\end{array}$ \\
\hline Routing 2' & $\begin{array}{l}1 \text { blanking, forging }-2 \text { drilling C } 336-1-3 \text { turning of outside round, face and chamfer }-4 \text { reaming, turning of other face and cham- } \\
\text { fer }-5 \text { reaming }-6 \text { cleaning parts }-7 \text { quench-aging }-8 \text { fine boring SV-1000A }-9 \text { finish milling SV-1000A }-10 \text { grinding outside } \\
\text { round }-11 \text { honing inside hole }-12 \text { magnetic particle testing and final checkout }-13 \text { cleaning, oil seal }\end{array}$ \\
\hline
\end{tabular}

Table 7. The evaluation results of the optimized process routings.

\begin{tabular}{|c|c|c|c|c|c|c|c|c|c|c|c|c|c|}
\hline \multirow{2}{*}{ Routings } & \multicolumn{12}{|c|}{ longitudinal Process Evaluation Value } & \multirow{2}{*}{$\begin{array}{c}\text { Comprehensive } \\
\text { Value }\end{array}$} \\
\hline & 1 & 2 & 3 & 4 & 5 & 6 & 7 & 8 & 9 & 10 & 11 & 12 & \\
\hline Routing 2 & 90 & 95.2 & 89.6 & 89.3 & 92.5 & 95.8 & 94.2 & 93.6 & 95.1 & 92.9 & 88.4 & 89.3 & 92.80 \\
\hline
\end{tabular}

Step 5: Evaluate the optimized routing 1' and routing 2' again and the comprehensive evaluation values are further improved to 94.18 and 92.8 , as shown in Table 7. Finally the optimized routing 1' is selected as the optimal process route.

\section{CONCLUSION}

The paper first puts forward the method to construct the green process databases for a specific product range and then proposes a local optimization method of green process based on process features on the foundation of the process routes being used to improve their practicability. When building green process databases, process features, processing schemes and related resources are focused on. With the support of green databases, Leonid Potter interaction matrix model is employed to evaluate and analyze the initial process routes to determine the process routes and the "problem" processes to be optimized, then local optimization based on process features is performed with ant colony algorithm to obtain the process route with good performance on green property. The designed method takes full advantage of the current process routes proved by many years' enterprise practice, so it could be operated more effectively and the results would be more convincing. Finally by an example, the proposed method is verified to be feasible, effective and practical. 


\section{CONFLICT OF INTEREST}

The authors confirm that this article content has no conflicts of interest.

\section{ACKNOWLEDGEMENTS}

The research is supported by the Technology Pillar Program during the 12th Five-year Plan Period of China under Grant No. 2012BAF01B01 and the National Natural Science Foundation of China (NSFC) under Grant No. 51205429, and the National Science.

\section{REFERENCE}

HE Yan, LIU Fei, CAO Hua-jun, ZHANG Hua. Process planning support system for green manufacturing and its application[J].Computer Integrated Manufacturing System, 2005, 11(7): 976-980.

[2] Huang Juan-juan, Wang Yong-chao, Wang Xiao-bing, Zhang Qun. Web-based green manufacturing process planning[J]. Machine Design \& Manufacture, 2011, 6(6): 246-248.

[3] BiKexin, Ma Huizi, Li Wanhong. Interpretive structural modeling of critical success factors for implementing green process innovation in manufacturing enterprises[J]. Advanced Materials Research: 2012, (361): 1026-1029.

[4] Cao Hua-jun, Liu Fei, He Yan, Zhang Hua. Study on model set based process planning strategy for green manufacturing. Computer Integrated Manufacturing System, 2002, 8(12): 978-1001.
[5] Jiang Zhi-Gang, Zhang Hua, Cao Hua-jun, He Yan. Process planning database research for green manufacturing $[\mathrm{J}]$. Process equipment, 2005,32(5): 42-45.

[6] Zhang Hua, Wang Xi-bin. Green assessment of manufacturing process based on the data envelopment analysis[J]. Ordnance Journal, 2005, 26(4): 523-527.

[7] TAN Xian-chun. Process elements and process optimization studies for green manufacturing process planning[D]. Journal of Chongqing University, 2004.

[8] WANG Xiao-bing, WANG Yong-chao, HUANG Juan-juan, ZHANG Qun. Process category selection and parameter optimization for green manufacturing[J]. Machine Design \& Manufacture, 2011, 10(10): 191-193.

[9] Tan Xianchun. A decision-making model of gear process for green manufacturing $[\mathrm{J}]$. Journal of Chongqing University, 2003(01).

[10] Tong Wei, Lei Qi. Research on green process route optimization method based on Fuzzy Synthetic and GA[OL].[2011-07-13]. Chinese Scientific Papers Online, http://www.paper.edu.cn/releasepaper/content/201107-188.

[11] Cao Hua-jun, Liu Fei, Yan Chun-ping, Li Cong-bo. Environmental impact evaluation method of manufacturing processes and its case[J]. Chinese Journal of Mechanical Engineering, 2005, 41(6): 163-167.

[12] Chen Xi-xiang, Qiu Jing, Liu Guan-jun. Research on the method of test-equipment selection based on analytic hierarchy process and fuzzy comprehensive assessment[J]. Acta Armamentarii, 2010, 1(31): 68-73.

[13] MENG Jiong, GUO Chun-Xiang, Li Sheng. Green process category selection method based on intuitionistic fuzzy cross entropy[J]. International Journal of Digital Content Technology and Its Applications, 2013, 7(1): 135-142.

Received: September 16, 2014

(C) Yuchuan et al.; Licensee Bentham Open.

This is an open access article licensed under the terms of the Creative Commons Attribution Non-Commercial License (http://creativecommons.org/licenses/by-nc/3.0/) which permits unrestricted, non-commercial use, distribution and reproduction in any medium, provided the work is properly cited. 